\title{
Vitrectomy and gas for inferior break retinal detachments: are the results comparable to vitrectomy, gas, and scleral buckle?
}

\author{
L Wickham, M Connor, G W Aylward
}

Br J Ophthalmol 2004;88:1376-1379. doi: 10.1136/bjo.2004.043687

\begin{abstract}
Aims: To compare the success rates of vitrectomy and gas with vitrectomy, gas, and buckle in the treatment of inferior break retinal detachments.

Methods: A retrospective case note review of 86 patients who presented with inferior break retinal detachments was carried out. An inferior break was defined as a horseshoe tear present between 4 and 8 o' clock. Patients were analysed in two groups; group A consisted of 41 patients who underwent a vitrectomy and gas, group B consisted of 45 patients who underwent a vitrectomy, gas, and scleral buckle. The features of the retinal detachment, peroperative and postoperative complications, and outcomes of treatment were recorded for each patient.

Results: The primary anatomical success rate at 3 months was $89 \%$ in group A versus $73 \%$ in group $B(p=0.11)$. There was no statistical difference in the complication rate between the two groups $(p=0.819)$. The most common cause of treatment failure was proliferative vitreoretinopathy, 20\% $(n=9)$ in group $B$ compared with $5 \%(n=2)$ in group $A$ and this reached statistical significance $(p=0.0159)$. There was a higher rate of epiretinal membrane development in group $B$ $(p=0.0004)$. The final attachment rate was not statistically different between the two groups, 95\% (39) in group A and $93 \%(42)$ in group $B(p=1.0)$.

Conclusion: Vitrectomy and gas without the application of a scleral buckle may be used to safely treat inferior break retinal detachments. It may be used as an alternative to vitrectomy, gas, and buckle which has an increased risk of choroidal haemorrhage, requires a longer operating time, and has all the associated complications of a scleral buckle.
\end{abstract}

$\mathrm{T}$ he use of vitrectomy and gas for the primary repair of rhegmatogenous retinal detachments has become increasingly accepted. ${ }^{1-3}$ A vitrectomy was initially reserved for patients in whom scleral buckling was thought to be difficult-for example, those with media opacities, posterior breaks, and multiple tears. ${ }^{4}$ However, over time more indications have been added and it is now the treatment of choice in many cases. ${ }^{1-3}$ When patients with inferior retinal break detachments require a vitrectomy it is routinely carried out in combination with a scleral buckle in order to support the inferior retina thus avoiding difficult posturing. This procedure is technically demanding, has an increased risk of choroidal haemorrhage, ${ }^{5}$ requires a longer operating time, ${ }^{6}$ and has all the associated complications of a scleral buckle-that is, exposure, refractive change, diplopia, possible decreased retinal blood flow, and risk of anterior segment ischaemia. ${ }^{7-12}$ A small case series by Tanner et al showed that buckling could be avoided in patients who had breaks in inferior retina. ${ }^{13}$ In this paper we review the results of patients with inferior break retinal detachments who underwent a vitrectomy without a buckling procedure. These results were compared with patients who underwent the standard treatment of vitrectomy, gas, and scleral buckle.

\section{METHODS}

A retrospective case note review of patients who were seen by the vitreoretinal service at Moorfields Eye Hospital was carried out. A vitreoretinal database was used to select all patients who underwent a primary vitrectomy with gas for a rhegmatogenous retinal detachment with one or more inferior retinal breaks from January 2001 to July 2003. An inferior break was defined as a horseshoe tear present between 4 and 8 o'clock. Exclusion criteria included previous retinal detachment surgery or vitrectomy, proliferative vitreoretinopathy (PVR) of grade $\mathrm{C}$ or greater, round hole detachments with no associated posterior vitreous detachment, giant retinal tears, retinoschisis, and paediatric cases. Once suitable patients were identified they were further subdivided into two groups. Group A consisted of patients who had a vitrectomy with gas and group B consisted of patients who underwent a vitrectomy with gas and scleral buckle.

Data collected on each patient included demographics, ocular co-morbidity, preoperative visual acuity, and clinical features of the retinal detachment, including type and distribution of all retinal breaks. All patients underwent surgery following an assessment by one of the five consultants in the vitreoretinal (VR) service and the on-call vitreoretinal fellow. The surgery was performed by a member of the on-call vitreoretinal team-that is, a registrar, fellow, or consultant. A standard three port vitrectomy was carried out using a wide angle viewing system (Oculus BIOM). Care was taken to relieve vitreous traction on all retinal tears. A lensectomy was only performed if the lens opacity was impairing the view during the surgery. Following the vitrectomy an internal search was carried out with 360 degree indentation. Additional retinal tears were treated with crotherapy or endolaser depending on their position. A fluid/air exchange was then performed via the most accessible retinal break. If there was no appropriate safe drainage site a retinotomy was performed. The primary break and the drainage site were treated with cryotherapy or endolaser as appropriate. Perfluorocarbon liquids were not routinely used as adjuncts to surgery. All patients underwent an air/gas exchange with either $\mathrm{SF}_{6}$ or $\mathrm{C}_{3} \mathrm{~F}_{8}$. Patients were postured either left, right, or alternate cheek to pillow depending on the distribution of the retinal breaks. Details of the surgical procedure and intraoperative and postoperative complications were recorded. The primary outcome

Abbreviations: PVR, proliferative vitreoretinopathy; VR, vitreoretinal 
Table 1 Preoperative characteristics. Group A consists of patients who underwent vitrectomy with gas and group B underwent vitrectomy, gas, and buckle

\begin{tabular}{llll}
\hline & Group A & Group B & p Value \\
\hline Ocular co-morbidity & $2(59 \%)$ & $24(53 \%)$ & 0.608 \\
None & $2(5 \%)$ & $6(13 \%)$ & \\
Amblyopia & $1(2 \%)$ & $2(4 \%)$ & \\
Glaucoma & 0 & $1(2 \%)$ & \\
Diabetic retinopathy & $2(5 \%)$ & $1(2 \%)$ & \\
Cataract & $7(17 \%)$ & $11(24 \%)$ & 0.877 \\
Pathological myopia & $5(12 \%)$ & 0 & \\
Other & $18(44 \%)$ & $19(42 \%)$ & \\
Lens status & $22(54 \%)$ & $24(53 \%)$ & \\
Phakic & $1(2 \%)$ & $2(4 \%)$ & \\
Pseudophakic & & & \\
Aphakic & &
\end{tabular}

of the procedure was then assessed at the 3 month postoperative visit. Data recorded at this stage included postoperative visual acuity, retinal status, and early postoperative complications. In those patients who redetached at this stage the number of operations required to successfully treat the detachment and the final outcome were also documented.

\section{RESULTS}

In all, 86 patients were included in the study; 41 (48\%) were assigned to group A and 45 (52\%) to group B. The average age was 60.0 years (SD 12.94).

The preoperative characteristics of the two groups showed no significant difference (0.608) (table 1).

In group A $25(61 \%)$ patients had horseshoe tears only, 16 $(37 \%)$ had a mixture of horseshoe tears and round holes. In $16(39 \%)$ patients the retinal breaks were confined to 48 o'clock (fig 1). The majority of patients $(22(54 \%))$ had a retinal detachment involving two quadrants, eight $(20 \%)$ involved three quadrants. Twenty (49\%) cases involved the macula. An associated vitreous haemorrhage was noted in four $(10 \%)$ patients.

Group B showed similar features; 29 (60\%) had horseshoe tears alone and $16(33 \%)$ had both horseshoe tears and round holes. These were confined to $4-8$ o'clock in $14(31 \%)$ patients (fig 2). Sixteen (36\%) patients had two quadrants of retinal detachment and in $15(33 \%)$ cases three quadrants of the retina were detached. There was no significant difference in the area of detachment between group A and group B $(p=0.206)$. Thirty $(67 \%)$ cases involved the macula. An associated vitreous haemorrhage was noted in three (7\%) patients. A $\chi^{2}$ test comparing the two groups showed no statistical difference $(\mathrm{p}=0.634)$.

In the scleral buckling group (group B) 17 (38\%) patients had one quadrant buckled, $17(38 \%)$ had two quadrants buckled, and 11 (24\%) underwent an encircling procedure; $93 \%$ (42) had uneventful surgery. There was one $(2 \%)$ case of lens touch, one (2\%) iatrogenic break, and one $(2 \%)$ retinal incarceration. There was no statistical difference in the complication rate when compared with group A

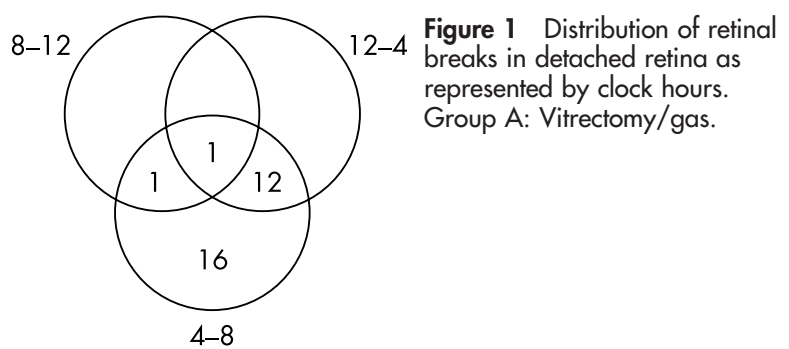

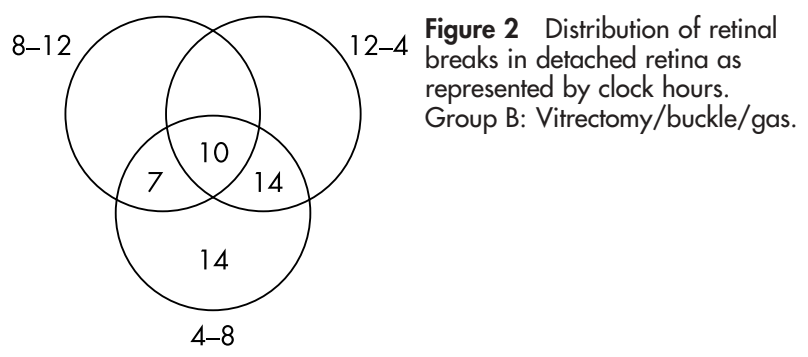

$(\mathrm{p}=0.819)$. In group A 95\% (39) patients had straightforward surgery and there was one $(2.5 \%)$ case of retinal incarceration and one $(2.5 \%)$ iatrogenic break.

The primary anatomic success rate was $89 \%$ in group A and $73 \%$ in group B ( $p=0.1102)$ (fig 3$)$. The most common cause of treatment failure in group B was PVR (table 2). The incidence of postoperative PVR was $20 \%(\mathrm{n}=9)$ in group B compared with $5 \%(\mathrm{n}=2)$ in group $\mathrm{A}$ and this reached statistical significance $(\mathrm{p}=0.0159)$.

The distribution of postoperative complications is shown in figure 4. Group B showed a higher rate of epiretinal membrane formation $(p=0.0004)$. Two patients in group $B$ developed a choroidal haemorrhage. In both cases this was not noted at the end of the operation but was observed at the postoperative check the following day.

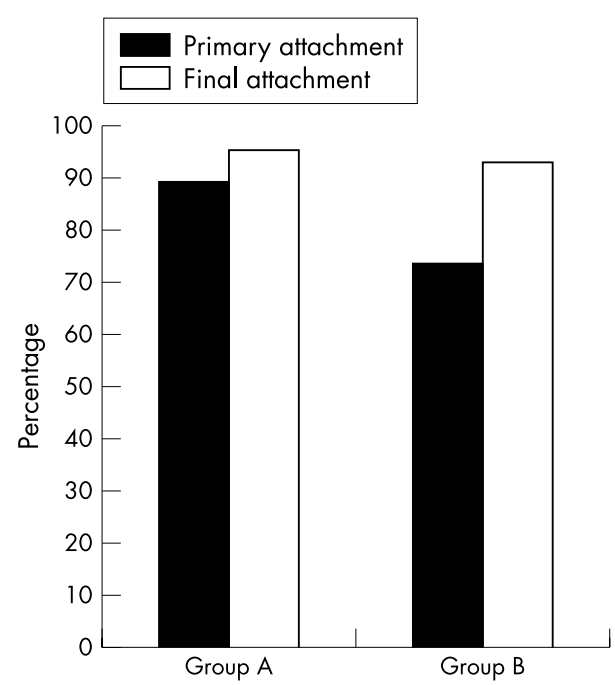

Figure 3 Retinal attachment rate following surgery. Group A: vitrectomy and gas, group B vitrectomy, gas, and buckle. 
Table 2 Causes of retinal redetachment at the 3 month postoperative visit and number of operations required to achieve retinal attachment

\begin{tabular}{llll}
\hline & Group A & Group B & p Value \\
\hline Reasons for failure & $1(2 \%)$ & $9(20 \%)$ & \\
PVR & $1(2 \%)$ & $2(4 \%)$ & 0.0159 \\
New break & $2(5 \%)$ & $1(2 \%)$ & 1.0 \\
Failure of treatment & $4(10 \%)$ & $3(7 \%)$ & 0.6034 \\
Number of operations required & $1(2 \%)$ & $5(11 \%)$ & 0.171 \\
1 & 0 & $2(4 \%)$ & \\
2 & & & \\
3 & & & \\
\hline
\end{tabular}

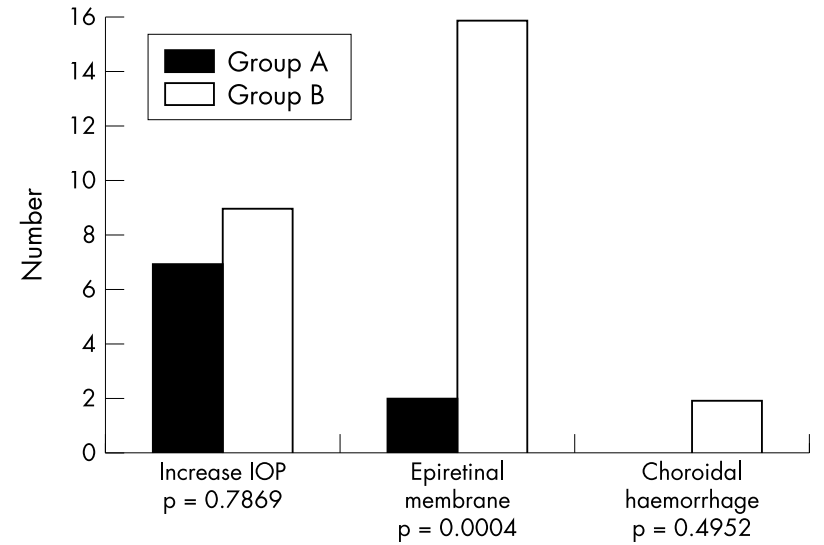

Figure 4 Postoperative complications. Group A: vitrectomy and gas, group B: vitrectomy, gas, and buckle.

The final attachment rate was not statistically different between the two groups, 95\% (39) in group A and 93\% (42) in group $B(p=1.0)$. Comparisons of the preoperative and postoperative visual acuity are given in figures 5 and 6 .

\section{DISCUSSION}

This study showed no significant difference between the final outcomes in patients treated with a vitrectomy and gas or a vitrectomy with gas and scleral buckle $(p=0.6)$. The decision to use a buckle in combination with a vitrectomy is usually taken in the presence of inferior retinal breaks or PVR. As mentioned earlier, this procedure is technically more demanding, has an increased risk of choroidal haemorrhage, ${ }^{5}$ requires a longer operating time, ${ }^{6}$ and has all the associated

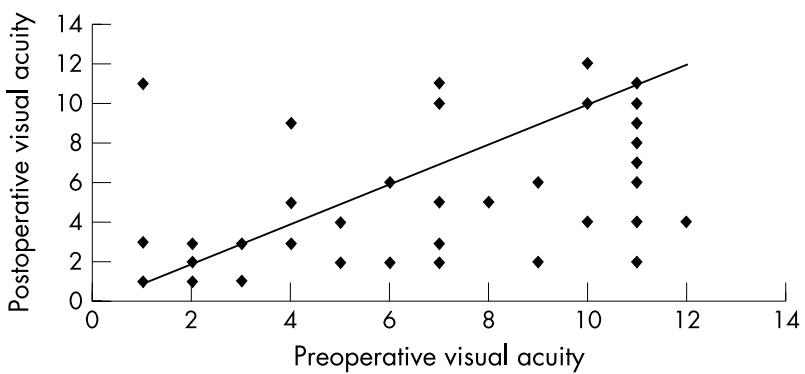

Figure 5 Scatter diagram comparing preoperative and postoperative visual acuities in group $A$. Key: $1=6 / 6,2=6 / 9,3=6 / 12,4=6 / 18$, $5=6 / 24,6=6 / 36,7=6 / 60,8=3 / 60,9=1 / 60,10=$ counting fingers, $11=$ hand movements, $12=$ perception of light, $13=$ nonperception of light. complications of a scleral buckle-that is, exposure, refractive change, diplopia, possible decreased retinal blood flow, and risk of anterior segment ischaemia..$^{7-12}$ The increased risks of this technique would be justified if it was associated with superior outcomes; however, the results of this study would suggest that this is not the case when treating inferior break retinal detachments. At present there is little published on the outcomes of vitrectomy and gas alone in these cases. Tanner et al published a prospective case series of nine patients showing a primary attachment rate of $8 / 9(89 \%)^{13}$ but the numbers are too small to draw any firm conclusions. Most other studies looking at the use of vitrectomy and gas for primary retinal detachment repair do not specify the position of breaks or the details of the operation performed. ${ }^{14}{ }^{15}$ Heimann et al carried out a retrospective study of 53 patients with rhegmatogenous retinal detachment. In their series the primary attachment rate was $64 \%$, but when these cases were subdivided according to position of the retinal breaks there was a $50 \%$ redetachment rate in the group with inferior breaks. ${ }^{4}$ This result would suggest a poor outcome for these patients; however, the numbers were small (six patients).

Although there is an absence of specific clinical evidence, there is published literature supporting the theoretical basis of this technique. The forces of retinal detachment and reattachment were well described by Machemer in $1984 .{ }^{16}$ In his Jacksonian lecture he showed that there were three factors required to achieve retinal re-attachment-that is, relief of traction, alteration of intraocular currents, and chorioretinal adhesion. All of these conditions can be addressed with a vitrectomy and gas. Relief of vitreous traction should be achieved during a vitrectomy; in fact, with the introduction of panoramic viewing systems-for example, oculus BIOM access to peripheral retinal pathology has been enhanced. ${ }^{17}$ Alteration of intraocular currents within the eye may be achieved with scleral buckling and with the

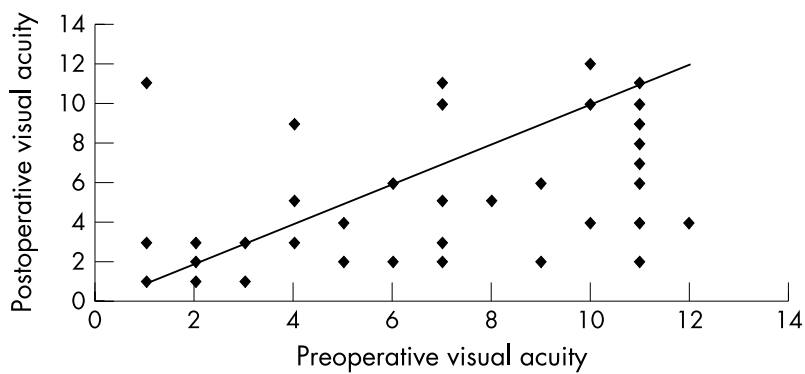

Figure 6 Scatter diagram comparing preoperative and postoperative visual acuities in group B. Key: $1=6 / 6,2=6 / 9,3=6 / 12,4=6 / 18$, $5=6 / 24,6=6 / 36,7=6 / 60,8=3 / 60,9=1 / 60,10=$ counting fingers, $11=$ hand movements, $12=$ perception of light, $13=$ nonperception of light. 
use of intraocular gases. ${ }^{16}{ }^{18} 19$ The introduction of air in the treatment of retinal detachment surgery dates back to work carried out by Ohm in $1911 .{ }^{20}$ Following the development of longer acting gases, the elucidation of their physical properties, and the increased use of vitrectomy, intraocular gases have become indispensable in the treatment of many vitreoretinal conditions. ${ }^{21-24}$ Based on this research it has been deduced that intraocular gases may not be used to tamponade inferior retinal pathology without awkward posturing or a scleral buckle. ${ }^{21-24}$ More recently, data have emerged showing that with a good intraocular gas fill the need for posturing following macular hole surgery can be reduced. ${ }^{25}{ }^{26}$ We have applied these findings to our practice to hypothesise that a good vitreous clearance combined with a large gas fill may allow us to treat inferior retinal pathology without the use of a scleral buckle.

In our study there was a significantly higher rate of PVR in group B (20\%) compared to group A (2\%). Although PVR was used as an exclusion criterion it is possible that these patients had undocumented preoperative PVR, which played a part in the choice of operation. All of the patients who developed PVR had a scleral buckle planned before the operation. This is one of the limitations of the retrospective nature of this study.

There was a significantly higher rate of epiretinal membrane formation noted in the postoperative examination of patients in group B which probably reflects the increased rate of PVR in this group.

There are a number of limitations to this study. Because of its retrospective nature no firm conclusions as to the relative merits of this technique compared with a vitrectomy and gas and buckle can be drawn. The surgery was also carried out by a number of surgeons, which allows for no consistent comparison of the results. This may in fact not be a significant disadvantage since our results would suggest that this approach is safe in the hands of a variety of surgeons of differing grades

In conclusion, this study suggests that there is a place for vitrectomy and gas without the use of a scleral buckle in the primary repair of patients with inferior retinal break detachments.

\section{Authors' affiliations \\ L Wickham, M Connor, G W Aylward, Western Eye Hospital, Marylebone Road, London NW1 5YE, UK}

Correspondence to: Louisa Wickham, Western Eye Hospital, Marylebone Road, London NW1 5YE, UK; lovisa.w@tiscali.co.uk

Accepted for publication 17 May 2004

\section{REFERENCES}

1 Leaver $\mathbf{P}$. Expanding the role of vitrectomy in retinal reattachment surgery. (Editorial) Br J Ophthalmol, 1993;77:197.

2 Gartry DS, Chignell AH, Franks WA, et al. Pars plana vitrectomy for the treatment of rhegmatogenous retinal detachment uncomplicated by advanced proliferative vitreouretinopathy. Br J Ophthalmol 1993;77:199-203.

3 Ah-Fat FG, Sharma MC, Majid MA, et al. Trends in vitreoretinal surgery at a tertiary referral centre: 1987 to 1996. Br J Ophthalmol 1999;83:396-8.

4 Heimann $\mathrm{H}$, Bornfeld N, Friedrichs W, et al. Pars plana vitrectomy without scleral buckling for rhegmatogenous retinal detachment. Graefes Arch Clin Exp Ophthalmol 1996;234:561-8.

5 Tabandeh H, Sullivan PM, Smahliuk P, et al. Suprachoroidal hemorrhage during pars plan vitrectomy-risk factors and outcomes. Ophthalmol 1999:106:236-42.

6 Hakin KN, Lavin MJ, Leaver PK. Primary vitrectomy for rhegmatogenous retinal detachment. Graefes Arch Clin Exp Ophthalmol 1993;231:344-6.

7 Findall RJ, Norton EW, Curtin, et al. Reduction of extrusion and infection following episcleral silicone implants and cryopexy in retinal detachment surgery. Am J Ophthalmol 1971;71:835-7.

8 Hayashi H, Hayashi K, Nakao F, et al. Corneal shape changes after scleral buckling surgery. Ophthalmology 1997;104:831-7.

9 Domniz Y, Cahana M, Avni I. Corneal surface changes after pars plana vitrectomy and scleral buckling surgery. I Cataract Refract Surg 2001; 27:868-72.

10 Fison PN, Chignell AH. Diplopia after retinal detachment surgery. Br J Ophthalmol 1987;71:521-5.

11 Kwartz J, Charles S, McCormack $\mathrm{P}$, et al. Anterior segment ischaemia following segmental scleral buckling. Br J Ophthalmol 1994;78:409-10.

12 Yoshida A, Feke GT, Green GJ, et al. Retinal circulatory changes after scleral buckling procedures. Am J Ophthalmol 1983;95:182-8.

13 Tanner V, Minihan M, Williamson T. Management of inferior retinal breaks during pars plana vitrectomy for retinal detachment. $\mathrm{Br} J$ Ophthalmol 2001;85:480-2.

14 Campo R, Sipperley J, Sneed S, et al. Pars plana vitrectomy without scleral buckle for pseudophakic retinal detachments. Ophthalmology 1999;106:1811-15.

15 Escoffery R, Olk J, Grand M, et al. Vitrectomy without scleral buckling for primary rhegmatogenous retinal detachment. Am J Ophthalmol 1985;99:275-81.

16 Machemer R. The importance of fluid absorption, traction, intraocular currents and chorioretinal scars in the therapy of rhegmatogenous retinal detachments. Am J Ophthalmol 1984;98:681-93.

17 Lesnoni G, Billi B, Rossi T, et al. The use of panoramic viewing system in relaxing retinotomy and retinectomy. Retina 1997;17:186-90.

18 Mester U, Anterist N, Kroll P, et al. The role of the vitreous in retinal detachment surgery with external buckling. Ophthalmologica 2002;216:242-5.

19 Clemens S, Kroll P, Stein E, et al. Experimental studies on the disappearance of subretinal fluid after episcleral buckling procedures without drainage. Graefes Arch Clin Exp Ophthalmol 1987;225:16-18.

$20 \mathrm{Ohm} \mathrm{J}$. Ueber die Behandlung der Netzhautablosung durch operative Entleerung der subretinalen Flussigkeit und Einspiritzung von Luft in den Glaskorper. Graefes Arch Clin Ophthalmol 1911;79:442-50.

21 Norton W. Intraocular gas in the management of selected retinal detachments. Trans Am Acad Ophthalmol Otolaryngol 1973;77:85-98.

22 Parver L, Lincoff H. Mechanics of intraocular gas. Invest Ophthalmol Visual Sci 1978;17:77-9.

23 Fawcett I, Williams R, Wong D. Contact angles of substances used for internal tamponade in retinal detachment surgery. Graefes Arch Clin Exp Ophthalmol 1994;232:438-44.

24 De Juan E, McCuen B, Tiedeman J. Intraocular tamponade and surface tension. Surv Ophthalmol 1985;30:47-51.

25 Simcock P, Scalia S. Phacovitrectomy without prone posture for full thickness macular holes. Br J Ophthalmol 2001;85:1316-19.

26 Tornambe P, Poliner L, Grote K. Macular hole surgery without face-down positioning. Retina 1997;17:179-85. 